\title{
Double Reverse Flow of Orbitary Vessels Following Mechanical Thrombectomy in Acute Ischemic Stroke
}

\section{Introduction}

Duplex ultrasound is a noninvasive and rapid technique that offers information on cerebral vascular hemodynamics in real time, which is very useful in acute stroke. Specifically, the orbital study provides important information on collateralization in severe cases of cervical carotid stenosis, showing an inversion of the ophthalmic artery (OA).

Less frequently evaluated, hemodynamic changes in the ophthalmic vein provide information on the presence of arteriovenous malformations or fistulas (Erickson SJ et al. Radiology 1989: 173 (2): 511-516).

We report a case of ophthalmic artery and vein inversion simultaneously after mechanical thrombectomy (MT).

\section{Case Report}

A 53-year-old woman with a past medical history of hypertension and dyslipidemia, with a favorable baseline situation (modified Rankin Scale/mRS 0), was transferred to a tertiary hospital after activation of stroke code due to sudden onset of aphasia and right hemiplegia. On arrival, she presented with symptoms compatible with left middle cerebral artery (MCA) stroke, with a National Institutes of Health Stroke Score (NIHSS) of $19(1 / 2 / 0 / 1 / 2 / 1 / 4 / 0 / 4 / 0 / 0 / 12 / 1 / 0)$. Cranial CT scan was performed and showed hyperdensity of the left MCA and loss of ipsilateral insular cortico-subcortical differentiation (ASPECTs 9), compatible with acute stroke in the territory of the left MCA. CT angiography revealed a tandem occlusion of the left internal carotid artery (ICA) and M1 segment of the left MCA. Treatment with ev alteplase was started (2:30 hours from the onset of symptoms) and mechanical thrombectomy was performed under general anesthesia (time door-to-needle/DTN 0:33 minutes). Catheterization of the right common carotid artery (CCA) was performed through right femoral puncture, revealing filling defects in left $M 1$, left $A 2$, and right $A 2$ (See - Fig. 1). After that, an $8 \mathrm{~F}$ introducer was placed in the left CCA, revealing an occlusion of the left ICA from its origin. The left ICA occlusion was crossed, and
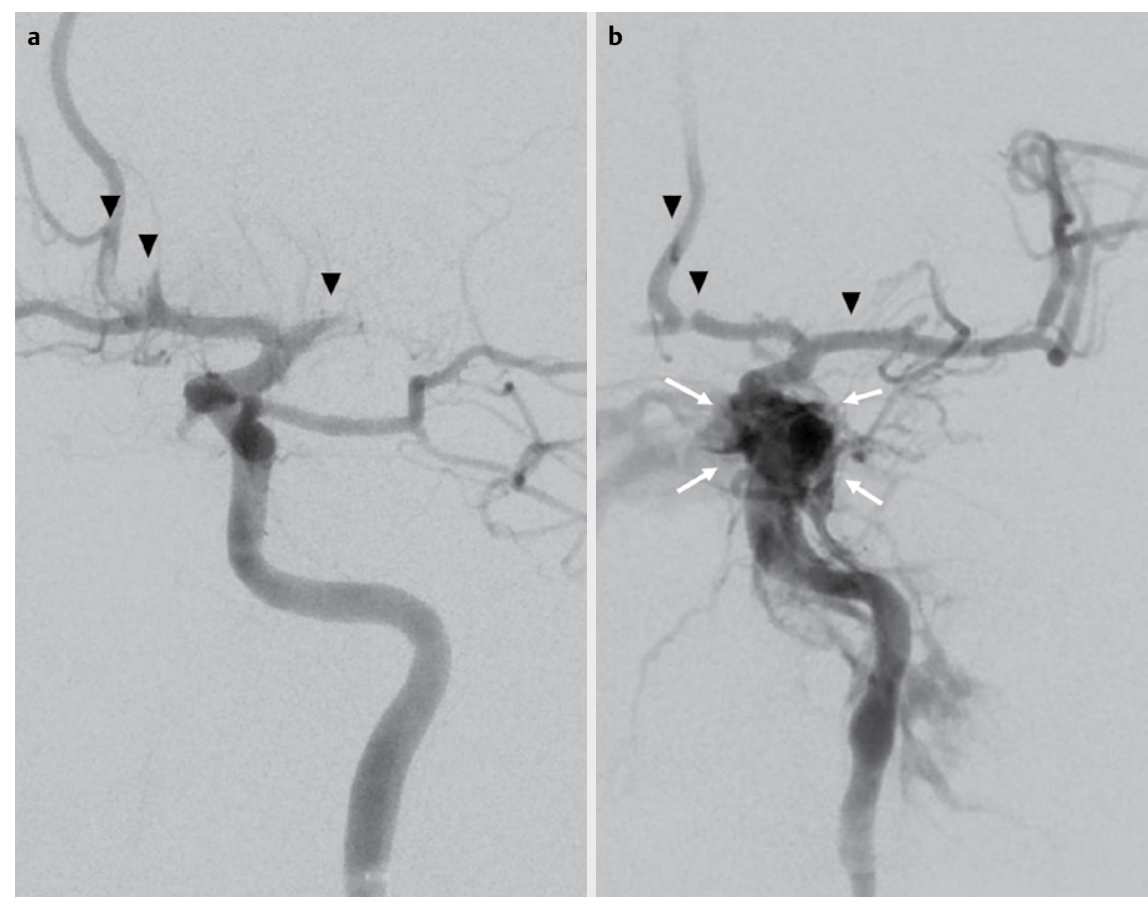

- Fig. 1 Digital subtraction angiography, AP view. a) Black arrows show the occlusion, from right to left, of the M1 segment of the left MCA, the A2 segment of the left ACA and the right A2. b) Black arrows show recanalization of the left $M 1$ and right $A 2$ segments, persisting thrombotic occlusion of left A2. White arrows show contrast leakage of the left ICA in the cavernous sinus due to iatrogenic laceration of the ICA, showing direct/high-flow left CCF.

an aspiration pass was performed in left M1, successfully revascularizing the left MCA circulation. An MT attempt was made to revascularize the bilateral A2 defects. The thrombus was impacted in the left anterior cerebral artery (ACA), leaving the right ACA permeable. The anatomy of the terminal left ICA-left A1 transition was hostile, resulting in failure of the aspiration catheter to advance to left A1-A2, causing a left ICA laceration that caused a left iatrogenic carotid-cavernous fistula (CCF) (See > Fig. 1). In summary, the occlusion of the left ICA and MCA was successfully revascularized, with persistent left ACA occlusion and non-significant left ICA atheromatous residual stenosis, on which acute stenting was not performed due to the degree of stenosis and the bleeding complication arising during the procedure.

After the procedure, the patient was extubated and admitted to the Stroke Unit. Two hours after the procedure, the patient presented a decreased level of conscious- ness, so a neurosonological urgent study was performed. The orbital duplex scan showed an inverted left superior ophthalmic vein (SOV), with arterialized flow and increased velocities (peak systolic velocity/ PSV of $43.3 \mathrm{~cm} / \mathrm{s}$ and end diastolic velocity/ EDV of $27 \mathrm{~cm} / \mathrm{s}$ ) compatible with the reported iatrogenic CCF (See $>$ Fig. 2). Furthermore, the left OA was identified with an inverted flow with a PSV of $44.5 \mathrm{~cm} / \mathrm{s}$ and an EDV of $19.7 \mathrm{~cm} / \mathrm{s}$ (see - Fig. 3), suspicious for reocclusion of the left ICA vs. critical residual ICA stenosis, which was confirmed by the carotid duplex scan (see - Fig. 4). The transcranial duplex scan showed a permeable left M1 with normal velocities due to adequate collateralization by the OA, anterior communicating artery complex (inverted ACA), and posterior communicating artery. An urgent cranial CT scan was performed and revealed an established infarct in the territory of the left MCA and left ACA, with mass effect with subfalcine herniation and deviation and col- 


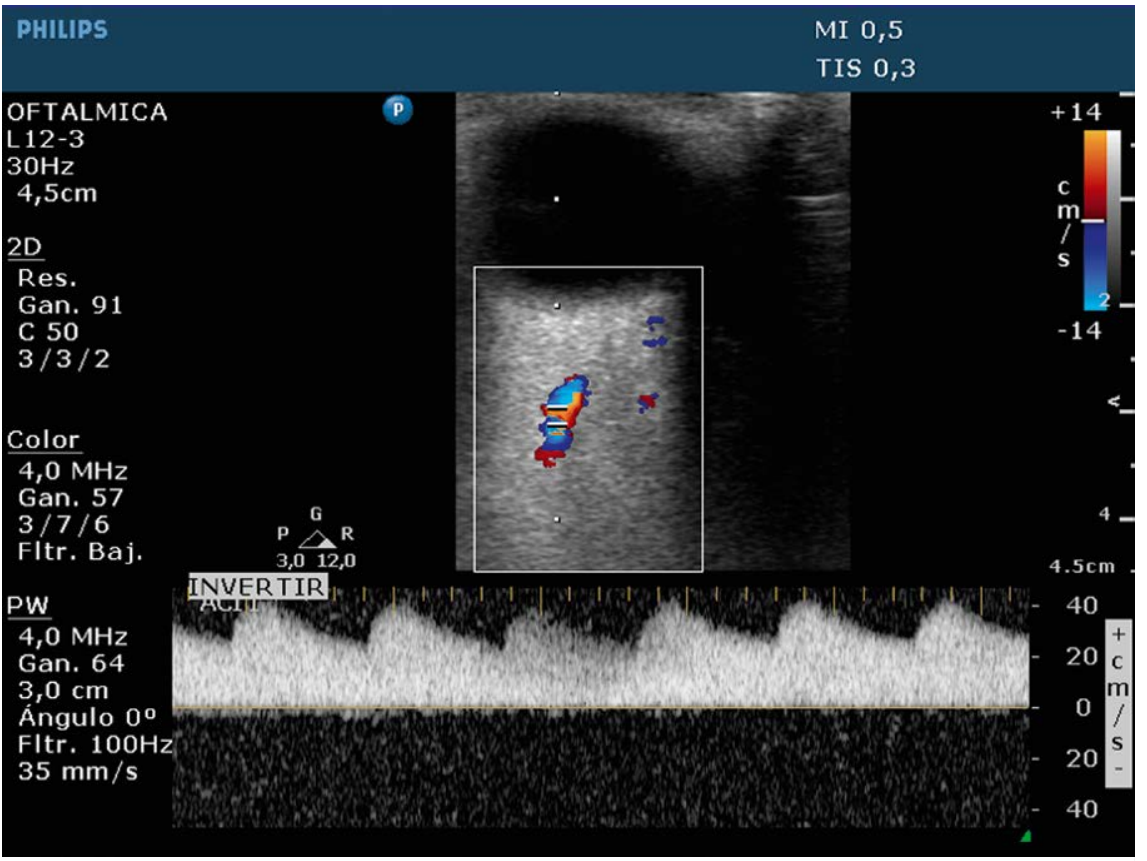

- Fig. 2 Orbitary duplex. The cursor points to the left superior ophthalmic vein, which is inverted and with an arterialized flow, compatible with carotid-cavernous fistula.

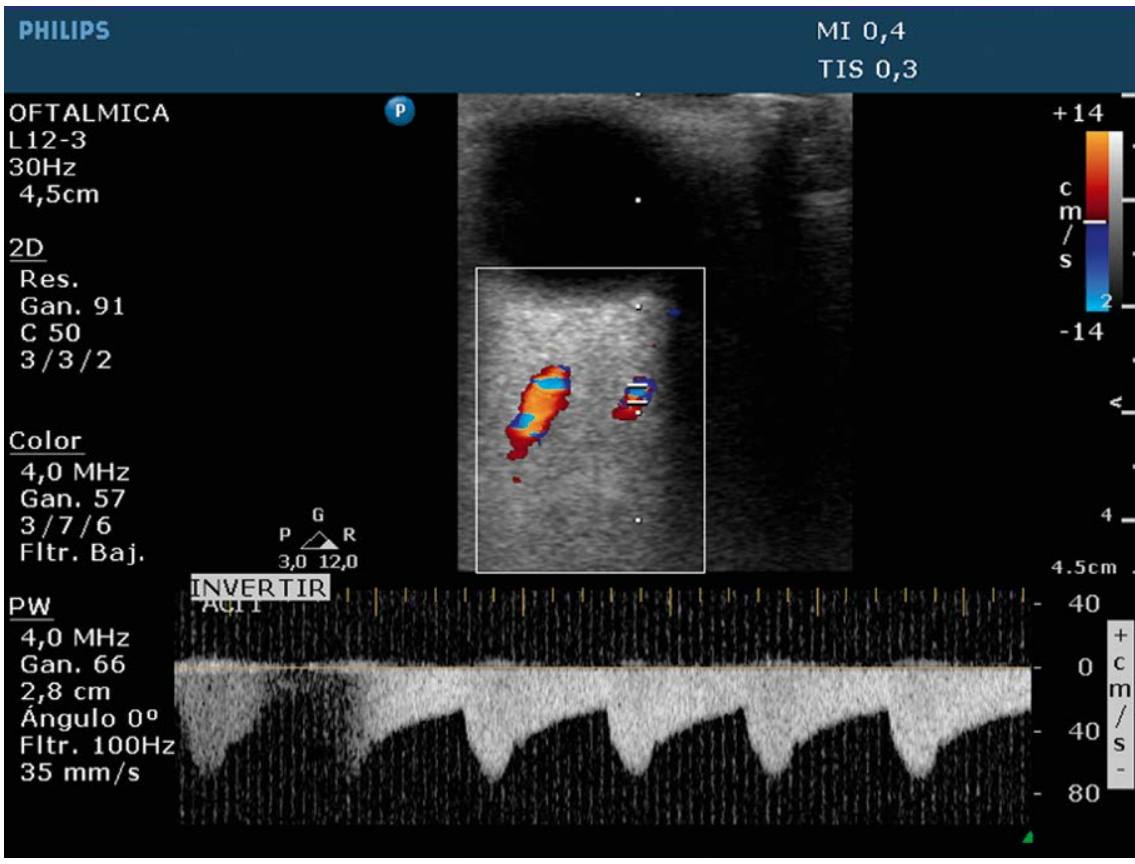

- Fig. 3 Orbitary duplex. The cursor points to the left ophthalmic artery, which is inverted due to occlusion of the left ICA. Medial to the ophthalmic artery, the superior ophthalmic vein is observed.

lapse of the left lateral ventricle, third ventricle, and partial right lateral ventricle, compatible with malignant infarction of the left MCA. The patient's family refused decompressive craniectomy. The patient presented subsequent neurological deterioration and finally died after 48 hours. has an anterograde flow with a PSV around $45 \mathrm{~cm} / \mathrm{s}$ and an EDV around $12 \mathrm{~cm} / \mathrm{s}$, which can be inverted in cases of high-grade carotid stenosis (Transquart $\mathrm{F}$ et al. J Clin UItrasound 2003; 31(5): 258-273), with a diagnostic sensitivity of $92.3 \%$ and a specificity of $100 \%$ (Erickson SJ et al. Radiology 1989: 173 (2): 511-516). It is important to identify the entire course of the artery, as the presence of loops can be misdiagnosed as flow reversal.

For its part, the SOV is located in a lateral position and superior to the OA. Under normal conditions, it presents a low pulsatility flow that moves away from the transducer, with a PSV around $10 \mathrm{~cm} / \mathrm{s}$. The reversal of this flow, as well as its arterialization with increased velocities and pulsatility (Transquart F et al. J Clin Ultrasound 2003; 31 (5): 258273; Duan Y et al. JUltrasound Med 2005; 24 (6): 739-745), are common findings described with high sensitivity (97\%) in cases of CCF (Duan Y et al. J Ultrasound Med 2005; 24 (6): 739-745).

As far as we know, this is the first case in the literature in which both findings (inversion of OA and SOV) occur simultaneously in a patient, highlighting two serious vascular complications, which we were able to detect early with the neurosonological study.

latrogenic CCF is a complication that can occur in up to $0.8 \%$ of transarterial endovascular procedures that involve the anterior cerebral circulation (Ono K et al. Interv Neuroradiol 2016; 22 (1): 91-96). Few cases related to MT in acute strokes have been reported and were usually associated with difficult procedures that required repeated passages with a stent retriever (Sheinberg DL et al. Cureus 2020; 12 (4): e7524). The first-line treatment for direct CCF is endovascular management (cavernous sinus embolization or placement of flow-diverter stents in the damaged ICA), since a low percentage of these tend to resolve spontaneously (Ono K et al. Interv Neuroradiol 2016; 22 (1): 91-96). In our case, we decided to postpone the treatment of the CCF to a second stage, based on the difficulty of the procedure due to the patient's vascular anatomy, in the patient thrombolyzed with alteplase.

Reocclusion of an atheromatous stenosis is another complication that can arise after MT. There is an increased risk associated with difficult procedures, athero- 


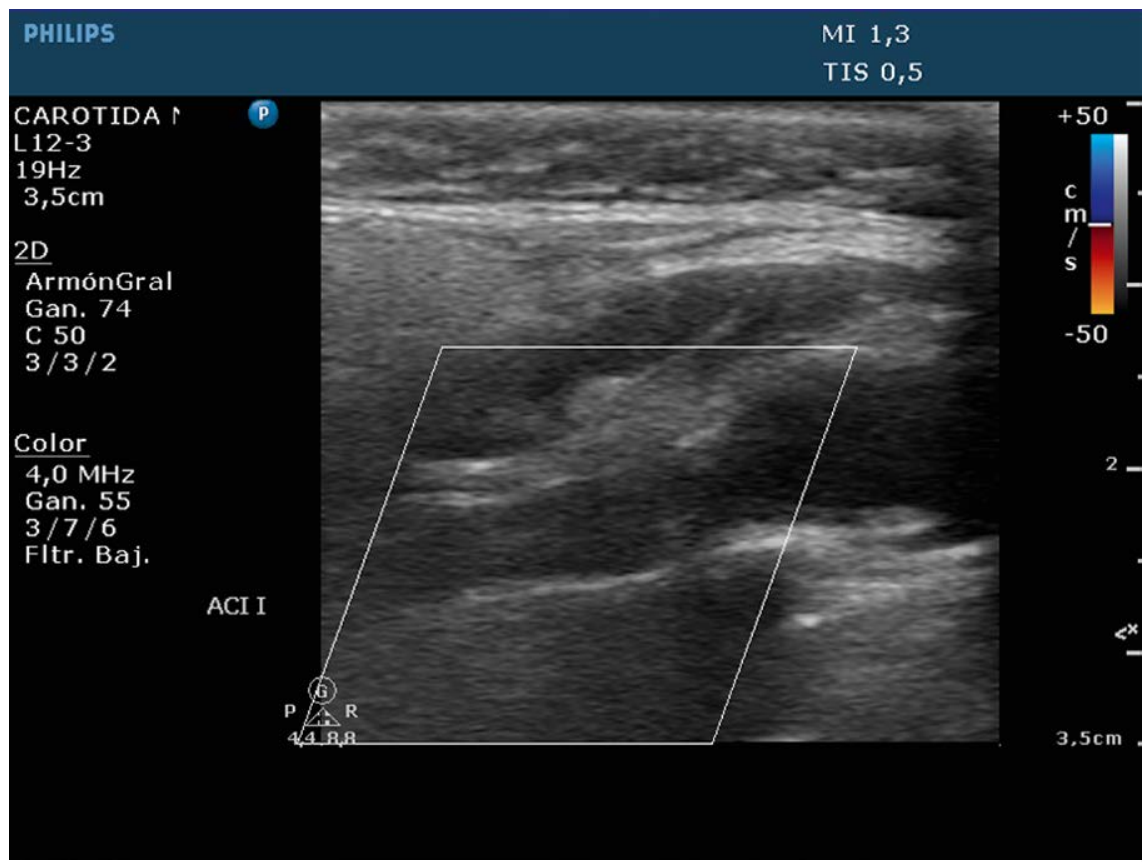

- Fig. 4 Carotid duplex with color. Left ICA is occluded from the beginning by hypoechoic heterogeneous material, with absence of flow inside.

thrombotic etiology, and residual stenosis after revascularization, among others.

In conclusion, orbital duplex is very useful in the neurosonological study of patients with acute cerebrovascular disease and can help in the detection of MT complications. A routine neurosonological study should be implemented in the early phase to detect possible complications.

\section{Conflict of Interest}

The authors declare that they have no conflict of interest.

Authors

Elena Elvira Soler ${ }^{(1)}$, Nicolás López Hernández, Isabel Beltran-Blasco, Blanca Serrano Serrano

\section{Affiliation}

NEUROLOGIA, Hospital General Universitar d'Alacant, Alicante, Spain

\section{Correspondence}

Dr. Elena Elvira Soler

Hospital General Universitari d'Alacant NEUROLOGIA

Pintor Baeza n¹1

03040 Alicante

Spain

Tel.: + 34/689/797 003

elenaelvirasoler@gmail.com

Bibliography

Ultrasound Int Open 2021; 7: E71-E73

DOI $10.1055 / a-1225-4388$

ISSN 2199-7152

(c) 2022. The Author(s).

This is an open access article published by Thieme under the terms of the Creative Commons Attribution-NonDerivative-NonCommercial-License, permitting copying and reproduction so long as the original work is given appropriate credit. Contents may not be used for commecial purposes, or adapted, remixed, transformed or built upon. (|s:|| creativecommons.org/licenses/by-nc-nd/4.0/) Georg Thieme Verlag KG, Rüdigerstraße 14, 70469 Stuttgart, Germany

\section{(ㄷ) (i) (으 $\Theta$}

